\title{
Topic Review
}

\section{Nanostructures GaAs solar cells}

\author{
Mohammad Nur-E-Alam ${ }^{1, *}$ and Narottam Das ${ }^{2,3}$ \\ 1 Electron Science Research Institute, Edith Cowan University, Perth, Australia; E-mail: \\ m.nur-e-alam@ecu.edu.au \\ 2 School of Engineering and Technology, Central Queensland University Australia, Melbourne, VIC 3000, \\ Australia. E-mail: n.das@cqu.edu.au (N. D.) \\ ${ }^{2}$ Centre for Intelligent Systems, School of Engineering and Technology, Central Queensland University, \\ Brisbane, QLD 4000, Australia. \\ * Correspondence: m.nur-e-alam@ecu.edu.au
}

Summary of entry: At present, the world is now passing a very far different time than normal situations due to the COVID-19 pandemic crisis. The global lifestyle and human civilization are currently progressing with down-stream that affecting almost every sector necessary for human civilizations except the current environmental situation. To control the COVID-19 spreading, most of the countries are following a lockdown process that reduces human mobility, thus reducing the $\mathrm{CO} 2$ emission to the environment. Though the COVID-19 pandemic is a blessing for the present environment, however, the post-COVID world will face a massive thrust of energy and only conventional energy resources may not be enough to mitigate the energy demands. Solar power generation technology mainly the photovoltaic (PV) systems and their advancement can be the leading possibilities to minimize the gap between power demand and generation. It is now time to think about how we can improve the PV power generation in the future and the post-COVID world. In this encyclopedia communication, we report on the Nano-technological approach to improving the conversion efficiency of GaAs solar cells. We have designed and optimized several types of nanostructured assemblies that can be implemented to reduce the front surface incident light reflection losses thus can assist to improve the conversion efficiency of GaAs solar cells.

Keywords: Conversion efficiency; GaAs substrate; light absorption; nano-grating structures; reflection loss; simulation; solar cells.

\section{Introduction}

The current world requires a huge demand for energy and the majority of that energy generation is mainly fossil fuels based. Also, to restraint the carbon dioxide (CO2) concentration in the air, it is time to think of suitable alternatives to fossil fuel-based energy generation and to minimize $\mathrm{CO} 2$ production. Photovoltaics (PVs) energy generation system definitely can provide an efficient and environmentally friendly solution for sustainable green earth among all the renewable energy resources. However, the global decarbonization goal reflects the need for innovative renewable technologies including the development and improvement of solar PV systems with other measures, such as proper business models and behavioral adaptation to reach zero emissions. Since the discovery of solar cells in the 19th century, they are widely used in various renewable energy generation projects including solar farms, roof-top installations, solar toys, solar windows, solar cars, solar mount street-light, portable solar battery bank systems, and spacecraft as well [1-3].

\section{Background, Motivation, and Performed work}

However, several factors that affect the performance of solar cells (i.e., mainly the conversion efficiency) including the structural defects to resistance to shading effects still pushing the scientists and researchers around the world to conduct research in this area for the development of various types of solar cells. The light reflection loss is one of the key features that the scientists are trying to 
improve for all types of solar cells. Different types of solar cells can absorb with different range of light spectra thus also vary the performance and conversion efficiency. However, all types of solar cells suffer less or more for the light reflection losses from its front surface. That is why various types of thin-film anti-reflective (AR) coatings are used to lower this reflection loss but these AR coatings also have limits to absorb a certain light spectrum. In addition, there are some disadvantages on using the AR coating, such as thermal and adhesive mismatch with the substrates, which requires a specific choice of material and geometry [4-7]. Figure 1 represents a glimpse of various solar cells, the basic concept, and relation of the light guiding towards light trapping into nano-structures.
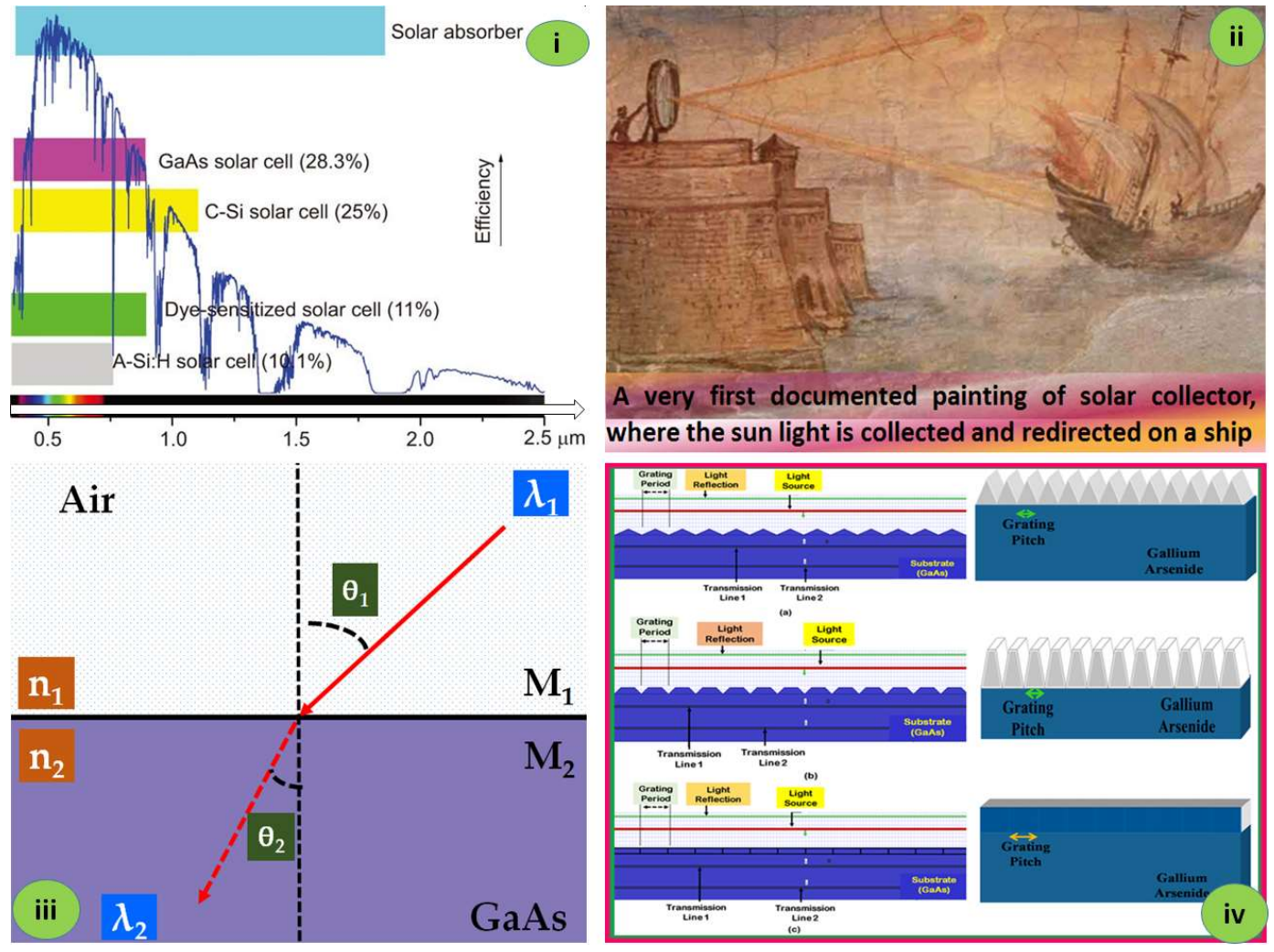

Figure 1. This figure is reproduced using the original sources published in References [1, 8, 9]; (i) the relative difference in efficiency of small-area various types of solar cells together with their light absorption range, (ii) a concept of manipulating and guiding the light into the desired direction, (iii) relation in between media-dependent angle of incidence and reflection, and (iv) schematic diagram for different types of nano-structured media helpful to engineer the light incidence reflection and trapping inside the nanostructures.

Due to the ability to make gradual changes in the refractive index the subwavelength grating (SWG) structures provide excellent antireflective and light trapping properties compared with a planar or flat type thin film. A significant number of research reports have been conducted worldwide and demonstrated using different types of nanostructures, such as diffractive nano-gratings, nanoholes, nanoparticles, double-gratings, and random textures for the enhancement of light absorption in solar cells [10-15]. The Finite-Difference-Time-Domain (FDTD) simulation tool is most commonly used to design, simulate, and analyze the properties of nano-grating structures. The computation is based on the principle of the FDTD method, i.e., mesh grid network, and Maxwell's equations. The Opti-wave FDTD simulation results, considering different nano-grating structures, such as triangular, trapezoidal (i.e., hatch top or truncated cone), and rectangular shapes are the main attraction of this encyclopedia entry. The nano-gratings height and aspect ratio are varied during the simulation process in order to find the best condition for capturing more incident light into the GaAs substrate for high conversion efficiency solar cells. A successful development of 
nanoscopic structure known as Moth's eye that helps to change the refractive index inside the nano-structure medium like the concept of nano-structured gratings and obtained the best light reflection coefficients of optimized nanostructured gratings are shown in Figure 2.
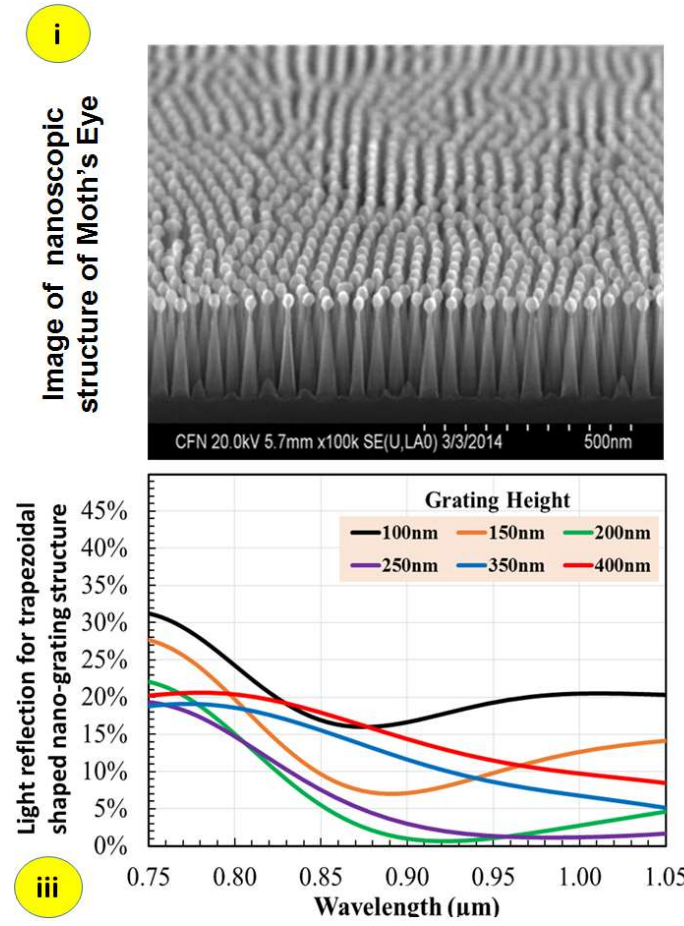
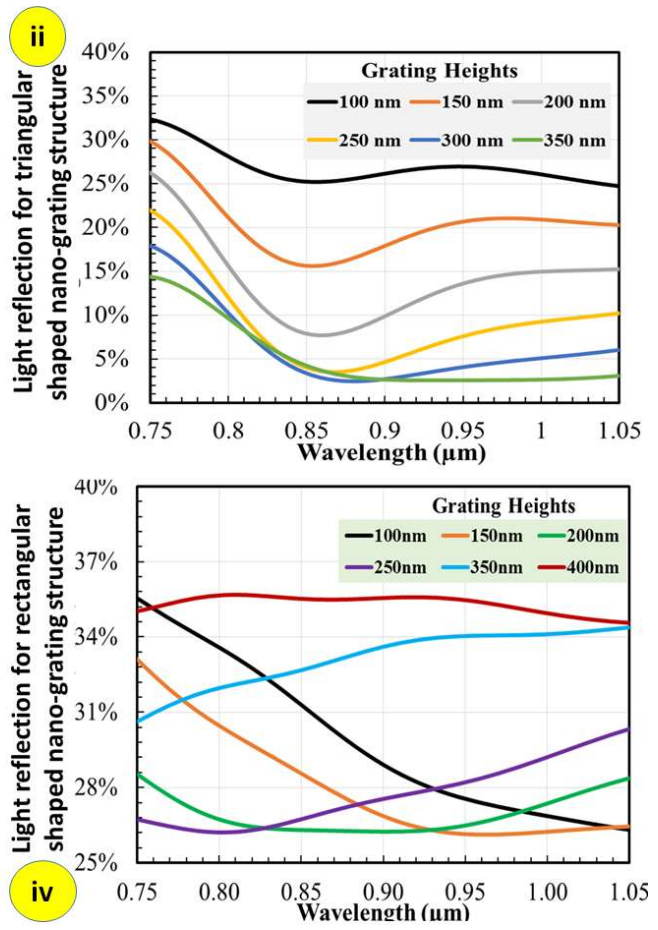

Figure 2. This figure is reproduced using the original simulated results published in Reference [1] together with the image of the nanoscopic structure of Moth's eye sourced out from the reference [16]; (i) image of the nanoscopic structure of Moth's eye, and (ii-iv) light reflection spectra obtained for triangular, trapezoidal and rectangular shapes of nano-gratings at different grating-height.

It is confirmed that the increase in nano-grating height leads to a reduction in light reflection, and this trend is satisfied for all nano-grating heights up to a certain range (height). From the simulation performance, we observed that the grating parameters played a significant role in the light absorption properties of the nanostructures as can be seen in Figure B (ii, iii, iv). In addition, with the increment of incident angle, the reflection of p-polarized light decreases until it reaches to the minimum reflection level at an angle of $75^{\circ}$ before it starts to rise again. However, the reflection of s-polarized light always increases for any angle of incidence. The light reflection of un-polarized remains at a constant stage from the beginning of $0^{\circ}$ to $60^{\circ}$ and then increased exponentially afterward as observed.

We have demonstrated and reported on the design, simulation, and analysis results of the light-capturing properties of nano-gratings with different shapes and geometries [1]. The optimized nanostructures that can be employed on top of the GaAs solar cells for the enhancement of the light absorption capacity into the solar cells and improving the conversion efficiency of the cells. It was evident from the simulation results that the triangular-shaped nano-grating structures enable us to absorb more lights into the GaAs substrate compared with other types of nano-grating shapes. The reason is that a series of consecutive reflections occur in the grooves (i.e., edges) of the inclined triangular walls and more energy localization happens due to the existence of sharp edges; moreover, using antireflective layer properties, it leads to several-step changes in the refractive indices of the grating geometry. These simulation results confirmed that the use of nano-grating structures enable up to $27 \%$ higher light absorption capacity than the conventional or flat type solar cells and thus can increase the conversion efficiency of GaAs solar cells. 


\section{Conclusions and Prospects}

We focus on the design and simulation of optimized nanostructured gratings suitable for the development of advanced GaAs solar cells with higher efficiency that can be a step forward towards a self-sustainable green globe.

Author Contributions: Conceptualization, N.D. and N.-E-A.; methodology, N.D.; software, N.D.; validation, N.D. and N.-E-A.; formal analysis, N.D. and N.-E-A.; investigation, N.D.; data curation, N.D. and N.-E-A.; writing-original draft preparation, N.D. and N.-E-A.; writing-review and editing, N.D. and N.-E-A.; visualization, N.D. and N.-E-A.; All authors have read and agreed to the published version of the manuscript.

Funding: This research received no external funding.

Conflicts of Interest: The authors declare no conflict of interest.

Entry Link: The Link to this entry published on the encyclopedia platform (https://encyclopedia.pub/item/revision/251bbd90f51d0379929efe66511a751e).

\section{References}

1. Das, N.; Chandrasekar D.; Nur-E-Alam, M.; Khan. M. M. K. Light Reflection Loss Reduction by Nano-structured Gratings for High Efficient Next-generation GaAs Solar Cells. Energies 2020, In Press.

2. IRENA's Global Renewables Outlook: Energy transformation 2050. https://www.irena.org/ publications/2020/Apr/Global-Renewables-Outlook-2020 (Accessed on 19/06/2020).

3. Andreev, V. M. GaAs and High-Efficiency Space Cells, Chapter-1, Practical Handbook of Photovoltaics (Second Edition), Fundamentals and Applications, 2012, 399-416.

4. Sagar, R.; Rao, A. Increasing the silicon solar cell efficiency with transition metal oxide nano-thin films as anti-reflection coatings. Mater. Res. Express, 2020, 7, 016433.

5. Mandal, P.; Sharma, S. Progress in plasmonic solar cell efficiency improvement: A status review. Renewable and Sustainable Energy Reviews, 2016, 65, 537-552.

6. Ateto, E. O.; Konagai, M.; Miyajima. S. Triple layer antireflection design concept for the front side of c-Si heterojunction solar cell based on the antireflective effect of nc-3C-SiC:H emitter layer. International Journal of Photoenergy, 2016, Article ID 5282851, doi.org/10.1155/2016/5282851.

7. Chanta, E.; Wongratanaphisan, D.; Gardchareon, A.; Phadungdhitidhada, S.; Ruankham, P.; Choopun, $\mathrm{S}$. Effect of $\mathrm{ZnO}$ double layer as anti-reflection coating layer in $\mathrm{ZnO}$ dye-sensitized solar cells. Energy Procedia, 2015, 79, 879-884.

8. Alapatt, G. Earth abundant thin film technology for next generation photovoltaic modules. PhD Thesis-2014, Clemson University.

9. Pastorelli, F. Light enhancements in nano-structured solar cells, Chapter 3, PhD Thesis-2013, Université Aix-Marseille, and Universitat Politécnica De Catalunya.

10. Rao, J.; Varlamov, S. Light trapping in thin film polycrystalline silicon solar cell using diffractive gratings. Energy Procedia, 2013, 33, 129 - 136.

11. Perl, E. E.; McMahon, W. E.; Bowers, J. E.; Friedman, D. J. Design of antireflective nanostructures and optical coatings for next-generation multijunction photovoltaic devices. Opt. Exp., 2014, 22 (S5), DOI:10.1364/OE.22.0A1243.

12. Lee, S.; In, S. J.; Mason, D. R.; Park, N. Incorporation of nanovoids into metallic gratings for broadband plasmonic organic solar cells. Opt. Exp., 2013, 21 (4), 4055-4060.

13. Quaranta, G.; Basset, G.; Martin, O. J. F.; Gallinet, B. Recent advances in resonant waveguide gratings. Laser Photonics Rev., 2018, 12, 1800017.

14. Elshorbagy, M. H.; Cuadrado, A.; Alda, J. Narrow absorption in ITO-free perovskite solar cells for sensing applications analyzed through electromagnetic simulation. Appl. Sci., 2019, 9, 4850; doi:10.3390/app9224850.

15. Ferry, V. E.; Polman, A.; Atwater, H. A. Modeling light trapping in nanostructured solar cells. ACS Nano, 2011, 5 (12), 10055-10064.

16. Matthew, W. Self-assembled nanotextures create antireflective surface on silicon solar cells. https://www.bnl.gov/newsroom/news.php?a=11685. (Accessed on 12/11/2015). 